\title{
A TLBO-BASED ENERGY EFFICIENT BASE STATION SWITCH OFF AND USER SUBCARRIER ALLOCATION ALGORITHM FOR OFDMA CELLULAR NETWORKS
}

\author{
Danial Davarpanah, Mohammad Reza Zamani, Mohsen Eslami and Taher Niknam \\ Department of Electrical and Electronics Engineering, Shiraz University of Technology, Iran
}

\begin{abstract}
Downlink of a cellular network with orthogonal frequency-division multiple access (OFDMA) is considered. Joint base station switch OFF and user subcarrier-allocation with guaranteed user quality of service, is shown to be a promising approach for reducing network's total power consumption. However, solving the aforementioned mix-integer and nonlinear optimization problem requires robust and powerful optimization techniques. In this paper, teaching-learning based optimization algorithm has been adopted to lower cellular network's total power consumption. The results show that the proposed technique is able to reduce network's total power consumption by determining a near optimum set of base stations to be switched OFF and near optimum subcarrier-user assignments. It is shown that the proposed scheme is superior to existing base station switch OFF schemes. Robustness of the proposed TLBO-based technique is verified.
\end{abstract}

Keywords:

Energy Efficiency, Green Cellular Network, Base Station Switching, Optimization, TLBO Algorithm

\section{INTRODUCTION}

Information and communication technology (ICT) indubitable has undergone extensive developments in all the social and economic fields of humanity so that the amount of accessibility and beneficial use of it plays a pivotal and decisive role. Due to the extreme increasing demand to use wireless communications, cellular networks, being an important part of ICT, has caused a substantial energy costs, and also serious effects on the intensification of carbon emissions and environmental problems. Over recent years' serious efforts have been dedicated to improve energy efficiency (EE) of cellular networks. The main target of commercial cellular network designers has traditionally been increasing capacity, data rate, diversity, robustness of services, and ubiquitous access. Nonetheless, EE of cellular networks has now become a decisive criterion. To take EE into consideration, especially in future cellular networks, a systematic change in devising wireless protocols and architectures, efficient redesigning of base stations (BSs), smart networks, cognitive radio and opportunistic network access, and use of relays and heterogeneous networks have been proposed [1] [2].

Along with the increasing demand for cellular communications technologies and phenomenal explosive growth of wireless devices and bandwidth-thirsty applications, the number of BSs to support such amount of data traffic and provide connectivity at any point with high data rates should increase at an astonishing rate. More than $80 \%$ of total cellular network power is consumed at radio access equipment of the network, especially BSs [3]. All BSs have dynamic and constant electrical energy usage. The BS's dynamic power is used for signal transmission. The constant part is used for operations such as signal processing, cooling, power supply, and backup battery charging [4]. Constant and dynamic power of a BS are dependent [5]. BS's Dynamic power has significant effect on the required power of power amplifier and cooling system of a BS. Fehske [5] has shown that by reducing the transmit power of a BS from $20 \mathrm{~W}$ to $10 \mathrm{~W}$, electrical power consumption of the $\mathrm{BS}$ is reduced from $766 \mathrm{~W}$ to $532 \mathrm{~W}$. The Fig. 1 shows power consumption of different BS sections.

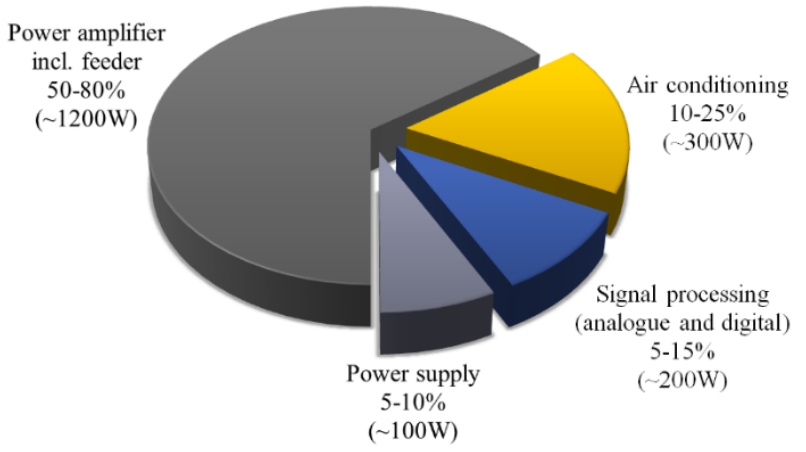

Fig.1. Power consumption of different BS sections [6]

In a cellular network, traffic load has significant fluctuations over time and space. There are two main reasons for traffic variations: 1) Typical user behavior during day and night. Usually there are more active users during day than night. 2) User mobility. Hence, there are time durations with off-peak traffic hours in any given area. With such traffic load variations, and in order to increase network's EE, the network should adopt itself to traffic load and reduce the number of active BSs in low traffic areas, while maintaining users' required quality of service (QoS). During the operation time of a BS, even when there is no traffic in the system, the BS consumes considerable amount of energy.

EE techniques addressing BSs power consumption reduction in a green cellular network are divided into two main categories [6]:

- Enhanced energy efficient hardware designs, such as CPU in digital processors, and power amplifiers.

- Enhanced energy efficient software design, such as enhanced algorithms that facilitate more efficient use of network.

This article presents new ideas and solutions that fall into the second category. The aim of this article is to present an energy efficient subcarrier allocation and BS switch OFF algorithm in downlink of an OFDM based cellular network. After dynamically switching OFF each low traffic BS, traffic is transferred to neighboring active BSs. However, there are two concerns that need to be addressed. One is that the neighboring BSs should have enough resources to accommodate new users. Second, the dynamic power of BSs should not increase. BS power increase is 
due to increase in distance, or equivalently path loss between remaining active BSs and users. Simultaneous search for BS candidates to be switched OFF and user subcarrier assignment is a complex mix-integer and nonlinear optimization problem, which is an NP-hard combinatorial problem, i.e., it combines the combinatorial difficulty of optimizing over discrete variable sets with the challenges of handling nonlinear functions, and does not have a trivial solution [8]-[10]. Therefore, wise use of metaheuristic optimization algorithms can give one low complexity near-optimum solutions. Considering the use of nonlinear optimization algorithms, genetic algorithms (GA) has been extensively used in solving wireless and cellular related problems [11]-[19]. In this work, teaching-learning based optimization (TLBO), which is a powerful nonlinear optimization technique, is adopted to find the best power efficient solution. The proposed Green Joint Successive Base Station Switch OFF and User Resource Allocation Optimization (GOSBOURAO) is shown to be very effective in reducing network's total power consumption with guaranteed user QoS.

The remainder of the paper is organized as follows. Related work is reviewed in section 2. System model and problem formulation are presented in section 3. The proposed BS switch OFF algorithm is presented in section 4 followed by simulations results in section 5. Finally, section 6 concludes the paper.

\section{RELATED WORK}

Extensive research has been carried out on improving BS performance in cellular networks, most of which have neglected power efficiency [20]-[23]. Nevertheless, recently compelling amount of research on reducing power consumption of fixed and mobile wireless networks has emerged [2] [24] [25]. Among these works, some only consider reducing the dynamic power of BSs [28]-[30]. Some others have considered BS's fixed power consumption as well, which are outlined as follows.

In [31] a power reduction algorithm based on BS sleep mode and using a fixed traffic pattern over time has been proposed. In [32], the authors considered daily traffic fluctuations and BS locations for the best BS switch OFF decision. Increasing BS deployment density to reduce path loss, and as a result reduce BSs dynamic power can be considered. However, as more BSs are deployed, total constant power usage of the network increases. The results presented in [32] show the impact of traffic load and BS density on sleep mode strategy and the resulting energy saving. Also, the authors in [33] study the effect of a number of several modes of switching BS to improve EE.

Samdanis [34] has proposed central and distributed algorithms for BS switch OFF. In the proposed central algorithm, at each stage, the BS with maximum traffic load is further loaded with neighboring BS's traffic. This strategy leaves some of the lowtraffic BSs without any traffic as good candidates to be switched OFF. Same idea is used in the distributed strategy, where coordination mechanism has been used to prevent network instability.

Suitable switching ON/OFF techniques for the BS have various form in response to active traffic demand variations. In [35], the authors propose a distance based BS switch ON/OFF algorithm in which, the mean distance between users and their serving BS in each cell is evaluated and the BS with highest average distances are selected to have priority for switching OFF. One of the important limitations that the distance based mechanisms are facing is that distance and path loss estimation errors are inevitable due to the fact doesn't exist very accurate path loss models or timing advance information that can be used to estimate the location and distances of user with respect to serving BS, which makes it a challenging problem.

In [36], the authors energy-efficient resource allocation multiuser orthogonal frequency-division multiple access (OFDMA) in a single cell with user QoS requirements has been studied. In [10], authors total network power consumption is minimized by switching BSs ON/OFF adaptively while maintaining network coverage. It is shown that BS activation problem for minimal network power consumption with full network coverage preservation is an NP-hard problem and a polynomial-time algorithm for energy-efficient BS activation is proposed. In [37], authors have considered practical and implementation aspects of BS ON/OFF switching; e.g., activation time and Ping-Pong effect.

Some recent works has gone toward the adjusting cell sizes and "cell zooming" techniques to reduce energy consumption. In [38] [39], the authors have introduced cell zooming, in which BSs adjust their coverage area according to network's circumstances or traffic in order to achieve traffic balance between cells, while reducing network's power consumption. An extreme case of cell zooming occurs when a cell's coverage area approaches zero, or in other words, the BS is switched OFF. This technique has the potential to disperse load for load balancing and concentrate load for reduce the energy consumption. However, there are some issues about these techniques that should be considered, including: In order to fulfill the objectives of cell zooming, traffic load fluctuations should be accurately traced and feed back to the cell zooming server, which that would be problematic if there were significant spatial and temporal fluctuations; Another issue that exists related to some of the cell zooming techniques is compatibility. Implementing almost many of the techniques of cell zooming require change to current structure of network management or for some reason are not supported by current cellular networks.

For minimizing power consumption of a cellular network, [40] has considered GA for network planning. Also in [41] BS sleeping strategies without affecting the desired user QoS for reducing network energy efficiency and using GA and particle swarm optimization (PSO) algorithms have been proposed. In addition to BS switch OFF, the amount of energy procured from different retailers, renewable energy and electricity retailers, is optimized.

In many previous works, the power reduction based on the sleep mode of the BSs is considered with regard to a fixed traffic period. This period is repeated at a specific time and BSs with active traffic load below the preset thresholds are switched OFF. This can include various disadvantages, such as, determining the suitable threshold is critical and challenging issue, which it has a great impact on the energy consumption of the network and the probability of blocking; Due to the traffic pattern is fixed, significant spatial fluctuations of users and traffic load are not considered; When a BS is switched OFF, all users are transferred simultaneously to the selected neighbor BS, which there is no certain optimality criteria for choosing the next BS to serve any user and can cause a lot of problems, including an inefficient 
increase network total power consumption, or if there are not enough resources to service these users at the next BS, which leads to blocking of a number of users. In fact, in many related work the main focus was to prioritize the switched OFF the BSs, then users were transferred together to the next specified BS. That's while the allocation for each user is not done separately according to the conditions in the current cell. However, the optimal allocation of users is extremely important in terms of reducing network's power consumption and switching ON/OFF of BSs must be coupled with user allocation. In proposed scheme, decisions to allocate users to the next BSs is done individually and resulted in a very high efficiency for the user allocation. It also does not require any special control channel to add or change the current network structure.

\section{SYSTEM MODEL AND PROBLEM FORMULATION}

\subsection{SYSTEM MODEL}

In general, the proposed scheme suits any cellular architecture with any number of connected cells. Without loss of generality, a cluster of cells in a conventional cellular network is considered in which, a central cell is surrounded by six hexagonal cells as shown in Fig.2. The 120 degree sectored antennas are assumed to have been deployed in each cell. BSs are located at the center of the cells and are assume to be connected through high speed backbone fiber to a central processing unit that allows them to exchange user link and traffic information. The number of active and switched OFF BSs at any given time is denoted by $N_{O N}$ and $N_{O F F}$, respectively.

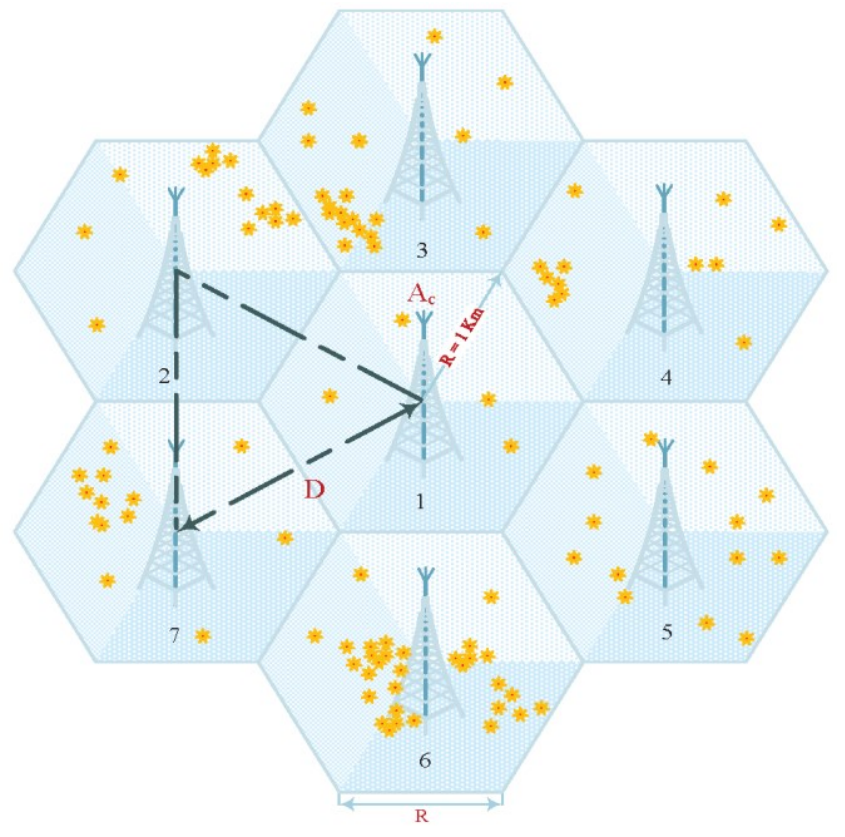

Fig.2. Cellular layout with $D=\sqrt{3} R$ and $A=\frac{3 \sqrt{3}}{2} R^{2}$

For the wireless channel, distance dependent path loss, lognormal shadowing and multipath Rayleigh fading are considered. The frequency channel tap between BS $b$ and user $u$ of subchannel $n^{c}$ is denoted by $h_{b, u, n}^{c}$. The total propagation loss $L(\mathrm{~dB})$ at distance $d(\mathrm{~m})$ is given by [42]: $L(\mathrm{~dB})=L_{0}+10 \beta \log _{10} d+X$, where $L_{0}=31.5 \mathrm{~dB}$ is assumed to be the path loss at $d=1 \mathrm{~m}$ and $\beta$ is the path loss exponent. $X(\mathrm{~dB})$, shadowing, is assumed to be a Gaussian random variable with zero mean and variance $\sigma_{X}^{2}(\mathrm{~dB})$. The average power consumption of a BS consists of a dynamic and a constant part, i.e., $P_{T}=P_{\text {dynamic }}+P_{\text {const }}$.

\subsection{PROBLEM FORMULATION}

Any large cellular network can be divided into clusters, each containing a number of neighboring cells. In this work, a cluster of cells is considered and it is assumed that all BSs within the cluster are operating and active. The aim is to find BSs that once they are switched OFF, cluster's total power consumption is reduced while user's QoS remain intact. In addition, the problem of re-assigning users that lose their connection once a $\mathrm{BS}$ is switched OFF to remaining active BSs is considered. Total power consumption is defined as

$$
P_{T}=\sum_{b=1}^{B} \sum_{u=1}^{U} \sum_{n^{c}=1}^{N^{c}} \gamma_{\left(b, u, n^{c}\right)} \alpha_{\left(b, u, n^{c}\right)}+\xi \sum_{b=1}^{B} \rho_{(b)}
$$

where $\gamma_{\left(b, u, n^{c}\right)}$ is the power required at BS $b$ that guarantees UE $u^{\text {th }}$ designated rate $q_{u}$ i.e., $\sum_{n^{c}=1}^{N_{L}} r_{\left(b, u, n^{c}\right)}=\sum_{n^{c}=1}^{N_{L}} \log _{2}\left(1+\frac{\left|h_{b, u, n^{c}}\right|^{2} \gamma_{\left(b, u, n^{c}\right)}}{\sigma_{n}^{2}}\right)$ $\geq q_{u}$, if sub-channel $n^{c}$ from BS $b$ is assigned to that user. $N^{L}$ is optimal number of subchannels calculated for each user. Furthermore,

$$
\alpha_{\left(b, u, n^{c}\right)}=\left\{\begin{array}{cc}
1 & \text { if channel } n^{c} \text { of BS } b \text { is assigned to } U E u \\
0 & \text { otherwise }
\end{array}\right.
$$

This indicates whether channel $n^{c}$ of BS $\mathrm{b}$ is assigned to UE $u$. Accordingly, the following mixed-integer nonlinear optimization problem needs to be solved:

$$
\min \left\{\sum_{b=1}^{B} \sum_{u=1}^{U} \sum_{n^{c}=1}^{N^{c}} \gamma_{\left(b, u, n^{c}\right)} \alpha_{\left(b, u, n^{c}\right)}+\xi \sum_{b=1}^{B} \rho_{(b)}\right\}
$$

subject to,

$$
\begin{gathered}
\sum_{u=1}^{U} \alpha_{\left(b, u, n^{c}\right)} \leq 1, b=1, \ldots, B ; u=1, \ldots, U \\
\sum_{b=1}^{B} \alpha_{(b, u)}=1, u=1, \ldots, U \\
\sum_{b=1}^{B} \sum_{n^{c}=1}^{N^{c}} \alpha_{\left(b, u, n^{c}\right)} \alpha_{\left(b, u, n^{c}\right)} \geq q_{(u)}, u=1, \ldots, U
\end{gathered}
$$

Constraint in Eq.(4) indicates that each subcarrier can be allocated to at most one user. For constraint Eq.(5) the following binary decision variable is introduced:

$$
\alpha_{(b, u)}= \begin{cases}1 & \text { if } \sum_{n^{c}}^{N^{c}} \alpha_{\left(b, u, n^{c}\right)}>0 \\ 0 & \text { otherwise }\end{cases}
$$


This shows whether any sub-channel of BS $b$ is assigned to UE $u$. Constraint in Eq.(5) indicates each user can only receive data from a single BS. Constraint in Eq.(6) indicates that sum of rates on all subchannels assigned from a BS to UE $u$ is greater than the designated rate (QoS) for that user, $q_{u}$, and shows whether a BS is switched ON and serves at least on UE or is switched OFF and serves no UE. All variables are listed in Table.1.

$$
\rho_{b}= \begin{cases}1 & \text { if } \sum_{u=1}^{U} \alpha_{(b, u)}>0 \\ 0 & \text { otherwise }\end{cases}
$$

Table.1. Parameter description of variables used in defining the problem

\begin{tabular}{|c|l|}
\hline Parameter & \multicolumn{1}{|c|}{ Definition } \\
\hline$B$ & Number of BSs in the cluster \\
\hline$U$ & Number of UEs in the cluster \\
\hline$N^{C}$ & Maximum number of sub-channels per BS \\
\hline$q_{(u)}$ & Data rate demand of UE \\
\hline$r_{\left(b, u, n^{c}\right)}$ & $\begin{array}{l}\text { Achieved rate when assigning channel } n^{c} \text { of BS } b \text { to } \\
\text { UE } u\end{array}$ \\
\hline$N_{E(b)}^{C}$ & $\begin{array}{l}\text { Number of empty sub-channels measured in each } \\
\text { BS }\end{array}$ \\
\hline$N_{O(b)}^{C}$ & $\begin{array}{l}\text { Number of occupied sub-channels by each UE at } \\
\text { related BS }\end{array}$ \\
\hline$\gamma_{\left(b, u, n^{c}\right)}$ & Power cost of assigning channel $n^{c}$ of BS $b$ to UE $u$ \\
\hline$\xi$ & Fixed power coefficient of a switched on BS \\
\hline
\end{tabular}

\section{PROPOSED GREEN JOINT SUCCESSIVE BASE SATATION SWITCH OFF AND USER RESOURCE ALLOCATION OPTIMIZATION (GOSBOURAO)}

To solve the nonlinear mix-integer programming optimization problem of Eq.(3), i.e., simultaneous search for BS candidates to be switched OFF and user subcarrier assignments that reduce network's total power consumption, one needs to find a suitable solution in the mature discipline of mathematical optimization. Such a complex mix-integer and nonlinear optimization problem is an NP-hard combinatorial problem, i.e., combines the combinatorial difficulty of optimizing over discrete variable sets with the challenges of handling nonlinear functions (for this case in the constraint domain), and does not have a trivial solution. Therefore, wise adoption of meta-heuristic optimization algorithms can give one low complexity near-optimum solutions.

There are many nature-inspired optimization algorithms [43][48] such as genetic algorithm (GA), particle swarm optimization (PSO), artificial bee colony (ABC), ant colony optimization (ACO), harmonic search (HS), the grenade explosion method (GEM), etc. The aforementioned algorithms have been used for many engineering problems and proven to be efficient in some cases. The GA is one of the most common evolutionary optimization technique that extensively used in solving problems. GA is used for complex problems with many variables and constraints. However, the use of the genetic algorithm and achieving a near optimal solution is simply not possible due to the proper tuning of the algorithm specific parameters. A change in the algorithm parameters affects the performance of the algorithm. In other words the effectiveness of the algorithm is influenced by each of these algorithm specific parameters and improper parameter adjustment either increases computations or yields a locally optimal solution instead of the global one [49] [50]. Similarly, it's about using the PSO, ABC, HS and many other useful optimization algorithms. Among these algorithms, TLBO [51] has proven to be an efficient optimization method for large scale nonlinear optimization problems in finding the global solution. TLBO does not require any algorithmic parameter adjustment, which gives it advantage over any other parameter dependent nonlinear optimization technique.

\subsection{TEACHING LEARNING OPTIMIZATION (TLBO) ALGORITHM \\ BASED}

TLBO is a new efficient optimization algorithm, which has been inspired by the influence of a teacher on learners in a class [51]. Here, learners' output is considered as their score or results. In this procedure, population consists of learners in a class and TLBO variables are courses provided. The teacher is generally considered as a highly educated person in a class who shares his or her knowledge with the learners in order to improve the output. The knowledge of the teacher affects the output of the students. It is obvious that a good teacher trains learners such that they can have better results (i.e., grades or marks). The level of knowledge of learners is evaluated by the mean value of the students' grade in classroom. The two fundamental components of this algorithm are Teacher and Learners. Based on two basic modes of the learning, through teacher (known as teacher phase) and interacting with the other learners (known as learner phase), TLBO procedure is divided into two parts, (1) Teacher phase and (2) Learner phase.

\subsubsection{Teacher Phase:}

The teacher efforts to enhance the mean knowledge of the class up to his or her own knowledge level. However, practically, this is limited by the learning capability of the class. Actually, in teacher phase, the level of knowledge of learners is affected by the good quality of the teacher as the best person. The teacher phase is formulated as follows:

$$
X_{n e w, i}^{k}=X_{i}^{k}+r\left(T_{h}^{k}-T_{f} M^{k}\right)
$$

where $T^{f}$ is the random valued learning factor vector with its $k^{\text {th }}$ element being 1 or 2 with equal probability. $T_{h}^{k}$ equals to the $X_{i}^{k}$, which results in the best cost function value. Optimization decision variables are contained in the vector $X_{i}^{k}$ and are replaced with $X_{n e w, i}^{k}$ only if they result in a superior cost function compared to that of $X_{i}^{k}$.

\subsubsection{Learner Phase:}

In this phase algorithm, as a meta-heuristic algorithm, the information is shared between the learners so that the level of their knowledge would be improved. A learner increase their knowledge by two different ways. One through the teacher phase and the other through the interact action with other learners. A 
learner learns something new if other learners have more knowledge than him or her.

This is described mathematically as:

for $i=1: N$

Randomly select two learners $X_{i}$ and $X_{j}$ where $i \neq j$

$$
\begin{aligned}
& \text { if } f\left(X_{i}\right)<f\left(X_{j}\right) \\
& \quad X_{\text {new }, i}^{k}=X_{i}^{k}+r\left(X_{i}^{k}-X_{j}^{k}\right)
\end{aligned}
$$

else

$$
X_{n e w, i}^{k}=X_{i}^{k}+r\left(X_{j}^{k}-X_{i}^{k}\right)
$$

end if

end for

Accept $X_{n e w, i}^{k}$ if it results in a superior objective function value.

Parameters in the aforementioned procedure are defined below,

- $k$ : Iteration index

- $i$ : Students index

- Number of the students in the class

- $r$ : A random variable in the range of $[0,1]$

- $X_{i}^{k}$ : Solution vector of $i^{\text {th }}$ student in $k^{\text {th }}$ iteration

- $X_{n e w, i}^{k}$ : New solution vector for $i^{\text {th }}$ student in $k^{\text {th }}$ iteration

- $T_{h}^{k}$ : Teacher in $k^{\text {th }}$ iteration

- $M^{k}$ : Mean class value in $k^{\text {th }}$ iteration

Proposed GOSBOURAO uses TLBO as its core optimizer and solves Eq.(3) with constraints given in Eq.(4)-Eq.(6). Vector $X_{i}^{k}$ contains all $\alpha_{\left(b, u, n^{c}\right)}$ decision variables. GOSBOURAO's steps are depicted in two flowcharts in Fig.3 and Fig.4. The steps of the first flowchart are described as follows:

Step 1: Set $i=1$. Assigned each user is to its serving BS. Initializing $\alpha_{\left(b, u, n^{c}\right)} \mathrm{s}$.

Step 2: Evaluate traffic load of each BS in terms of required number of OFDM sub-channels. Number of required sub-channels is obtained based on the capacity of each sub-channel and each user's requested QoS in terms of bit rate.

Step 3: Evaluate network's total power consumption, $P_{T}^{(i)}$, including constant and dynamic powers.

Step 4: Select the BS with minimum traffic load as the candidate to be switched OFF.

Step 5: Prepare a list of unoccupied sub-channels for each BS. This list is used for accommodating users once a BS is switched OFF. This list is also used to construct the initial population $X_{i}^{k} s$ for TLBO.

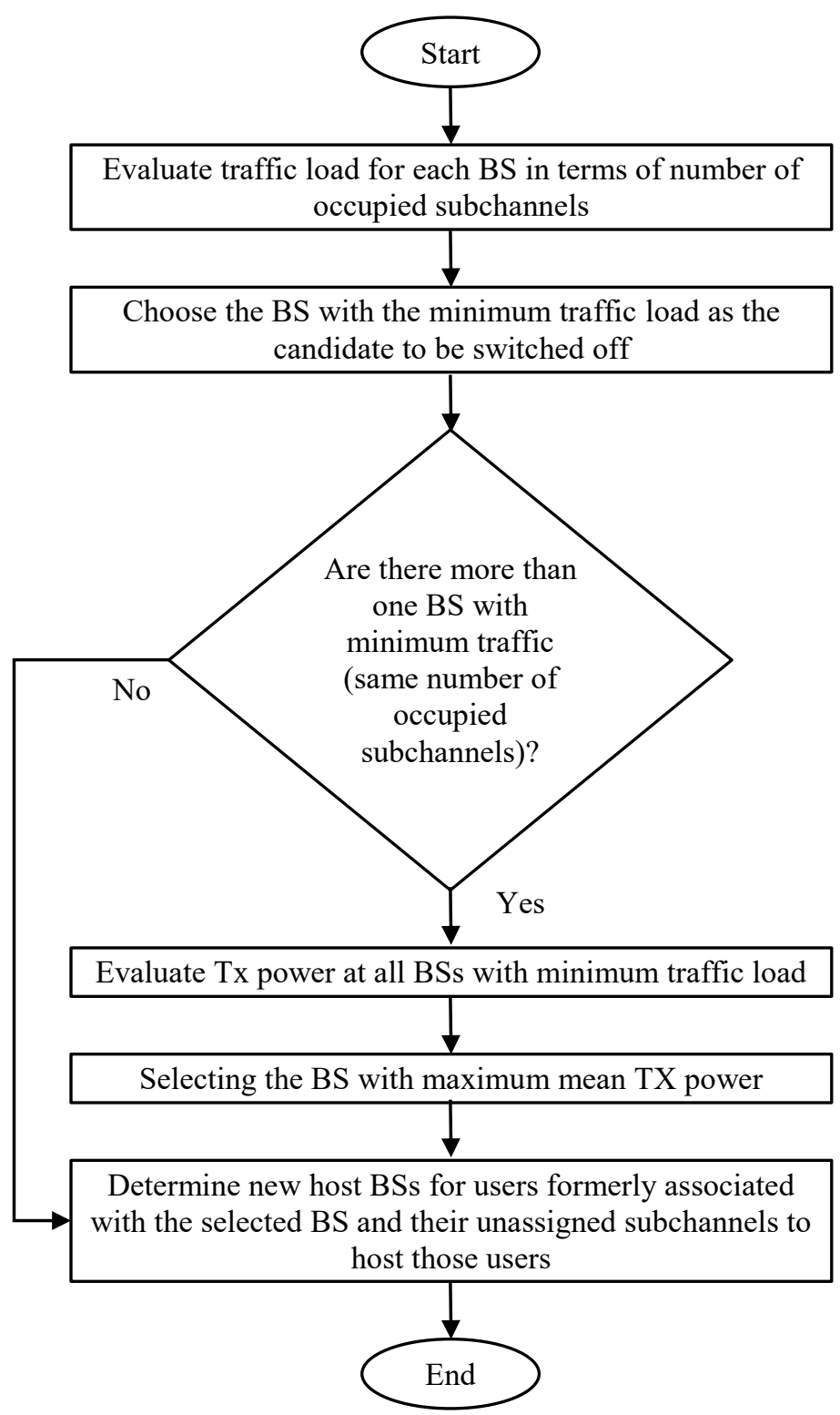

Fig.3. Flowchart of the proposed GOSBOURAO algorithm, Part 1: Finding the BS to be switched OFF

The steps of the flowchart of Fig.4 are described as follows:

Step 1: Execute TLBO to find the optimum sub-channel assignment for users of the candidate BS found in Step 4 of previous flowchart.

Step 2: Evaluate the updated total power consumption $P_{T}^{(i+1)}$ using results of Step 1 and assuming the candidate BS has been switched OFF.

Step 3: If $P_{T}^{(i+1)}<P_{T}^{(i)}$, terminate the algorithm.

Step 4: Re-assign the associated users of the selected BS to the remaining active BSs according to TLBO's output solution.

Step 5: Switch OFF the candidate BS. If $i=B-1$ terminate, otherwise $i=i+1$ and start over the whole process.

In the following section, performance of the proposed algorithm in terms of reducing network's total power consumption evaluated using simulation results. 


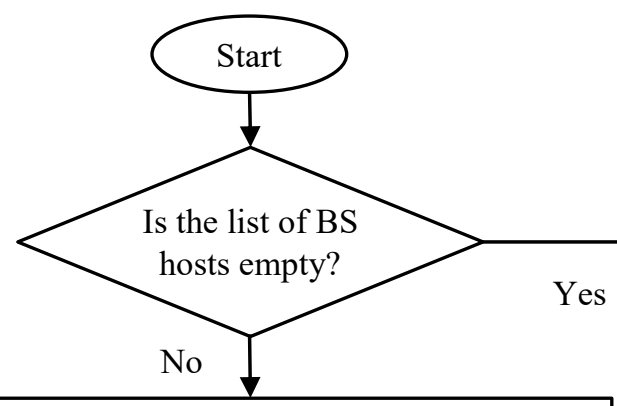

Use TLBO for subchannel to user assignment and network's total power consumption minimization

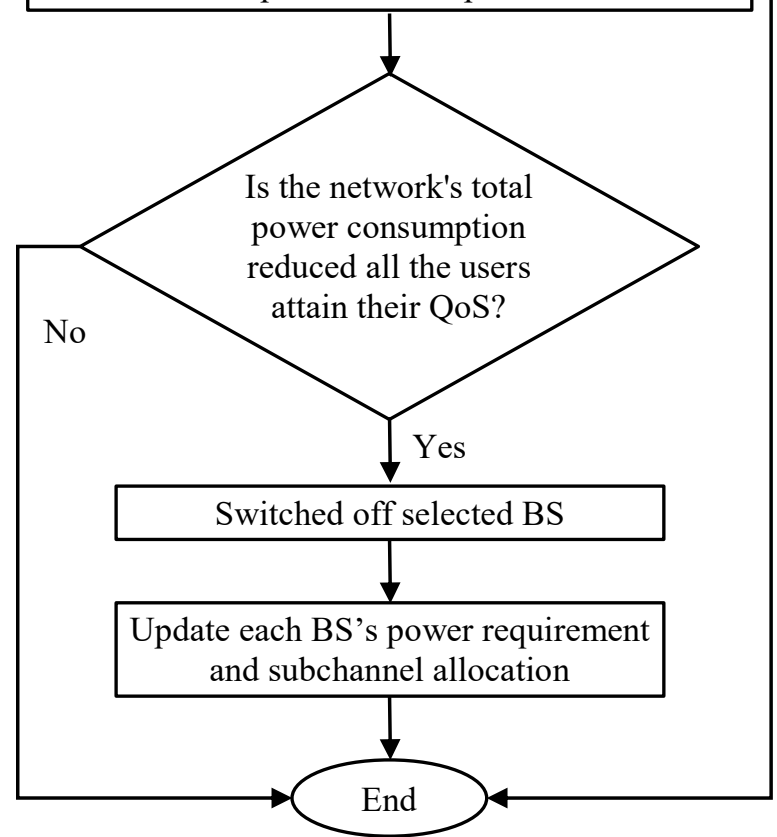

Fig.4. Flowchart of the proposed GOSBOURAO algorithm, Part 2: Sub-Channel Assignment

Table.2. System-level Simulation Parameters

\begin{tabular}{|c|c|c|}
\hline \multicolumn{3}{|c|}{ System Parameters } \\
\hline Parameter & Symbol & Value \\
\hline BS Maximal TX Power & $P_{\mathrm{TX} \text { max }}$ & $40 \mathrm{~W}$ \\
\hline System Bandwidth & $W_{S}$ & $20 \mathrm{MHz}$ \\
\hline UE Required Data Rate & $R_{U E}$ & $122,256,512(\mathrm{Kbps})$ \\
\hline Cell Radius & $r_{\text {cell }}$ & $1 \mathrm{Km}$ \\
\hline Number of Cell-sites & $N_{\text {cell }}$ & 7 \\
\hline $\begin{array}{c}\text { Cellular layout hexagonal } \\
\text { grid }\end{array}$ & - & 3 -sector sites \\
\hline Path loss Exponent & $\beta$ & 2.5 \\
\hline $\begin{array}{c}\text { Shadowing Standard } \\
\text { Deviation }\end{array}$ & $\sigma_{\text {shadow }}$ & $8 \mathrm{~dB}$ \\
\hline Constant Power of BS & $P_{\text {const }}$ & $600 \mathrm{~W}$ \\
\hline User Distribution & - & Uniform \\
\hline Subcarrier Spacing & $W_{C}$ & $20 \mathrm{kHz}$ \\
\hline
\end{tabular}

\section{SIMULATION RESULTS}

To evaluate the performance of the proposed scheme, a network consisting of a cluster of 7 macro BSs as described in section 3.1 is considered. The system parameters are summarized in Table.2.

Most of the strategies of switching ON/OFF that in order to response to active traffic demand variations have been proposed generally are load based approaches or distance based approaches. In order to compare the performance of proposed scheme, we implementation three different methods to determine which BS should be switched OFF. The first method is randomly switching OFF BSs with low traffic loads which this method mainly applies to BSs when their presence is not essential for the proper operation of the network and where few users are active. This method randomly switches some BSs to reduce energy consumption and radio coverage and services are taken care of by the cells that remain active. The second method is a greedy-based BS switch OFF algorithm [26] [34] which enforces BSs with higher traffic loads to serve more users and fills it with the traffic load of the neighboring BSs, which result in switches OFF BSs with no traffic load. The third method is based on the distance between the user's and their serving BS. In distance based sleepmode algorithm [35] required transmission power of BSs for serving users depends on the distance between users and BSs. In fact, the longer the distance indicative more transmission power needed in order to responsiveness users minimum service essentials. Accordingly, the distance between the user's and their serving BS can be an indicator of the energy saving and power consumption of cellular networks, so that the shorter the average distance between the users and BSs, the higher the energy saving. Hence, the distance based sleep-mode algorithm tends to switch OFF BSs with the longest distance between the users and BS to improve the energy saving of cellular networks.

The Fig. 5 shows clusters total power consumption at each stage of the proposed GOSBOURAO. The results have been obtained by averaging network's total power consumption, $P_{T}$, over 1000 network realizations. As Fig.5 shows, by switching OFF each BS, total power consumption of the network is reduced. Note that at each time the algorithm is executed, the optimal number of BSs that need to be switched OFF/remain switch ON will be decided. It can be seen that in the fourth stage of implementation of algorithms, the difference from the average power consumption of the entire network between the proposed algorithm and the distance based sleep-mode algorithm after 1000 network realizations is about $650 \mathrm{~W}$. For the 6 th $\mathrm{BS}$, the power reduction is incremental as almost the same amount of power that is saved in $P_{\text {const }}$ term by switching OFF a BS, is added to $P_{\text {dynamic }}$ term of active BSs to compensate the distance-based channel path loss.

This power trade-off can be better seen in Fig. 6 in the form of bar-chart where cluster's average total power increases after switching OFF the sixth BS. Here, GA and PSO based BS switch OFF, a distance based sleep-mode algorithm, a greedy-based BS switch OFF algorithm and the randomly switching BSs OFF one at a time have been compared with the proposed TLBO-based GOSBOURAO scheme. 


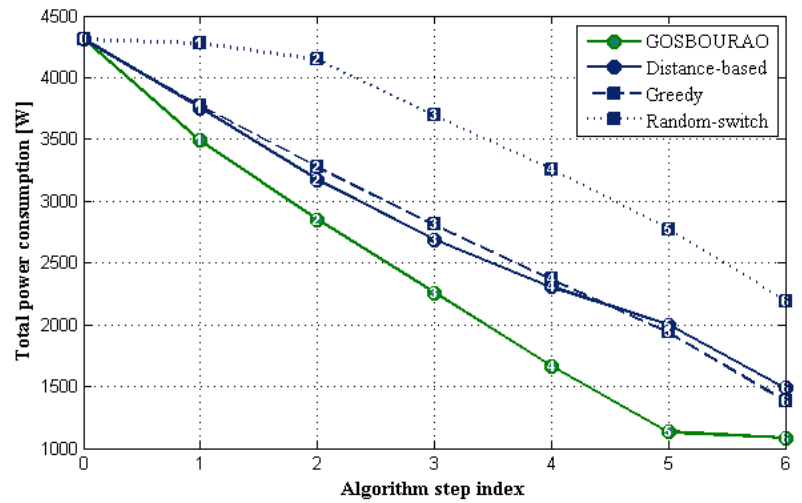

Fig.5. Total power consumption comparison of different BS switch OFF techniques

In addition, it can be seen that cluster's total power consumption has a minimum value at the fifth stage of BS GOSBOURAO for its powerful optimization core (TLBO) is able to better allocate sub-channel to users and reduce cluster's power consumption (switch OFF). Total power's increase at the last stage of the algorithm is due to the increased distances of some of the users to the last active BS in the cluster. Now, if we have the results of Fig. 6 as the output of the algorithms, in other words, only once the algorithms are executed and the results of Fig. 6 are considered, in the practical state when in the sixth step the power consumption of the entire network is greater than the previous one, according to proposed algorithm sixth BS will not be switched OFF and the number of optimal BSs to be switched OFF for this network is 5. Notable point regard to the implementation of algorithms is that in many scenarios, other implemented algorithms for a variety of reasons, including inappropriate allocation of users, cannot switches OFF the number of BSs and save energy as much as the proposed algorithm, and in the second or third stage they are not able to switches OFF another BS, which cannot be seen in the simulations.

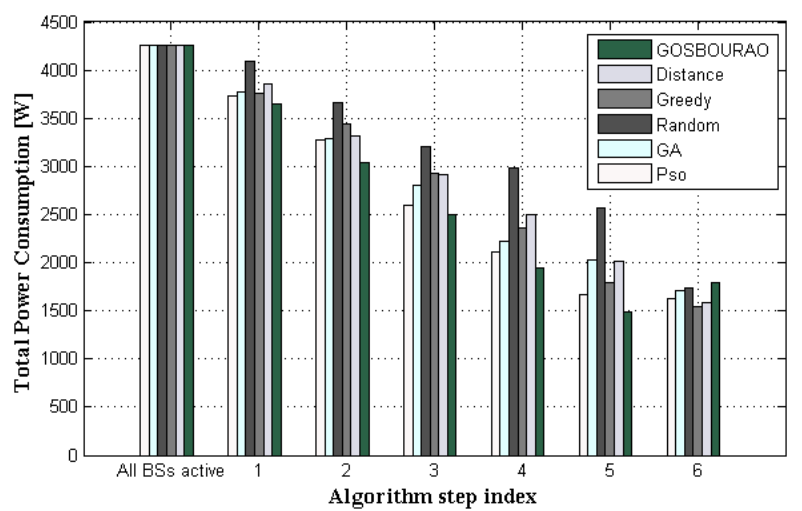

Fig.6. Bar chart power consumption comparison of different BS switch OFF techniques

\subsection{RELIABILITY OF THE PROPOSED GOSBOUROA}

In Table.3, total power consumption of the cluster when two BSs have been switched OFF, and the search for third candidate for switch OFF is going on (third stage) has been considered. The total power resulting from each optimization procedure execution has been considered as a random variable and its average, minimum, maximum and standard deviation $\left(E\left\{P_{T}[i]\right\}\right.$, $\min \left(P_{T}[i]\right), \max \left(P_{T}[i]\right)$ and $\left.\sigma\left(P_{T}\right)\right)$ values have been obtained. 50 runs have been used to generate 50 random samples over each channel realization. The results show that TLBO-based GOSBOURA has the minimum average and standard deviation, which means it is more reliable and energy efficient compared to the proposed scheme with GA/PSO core, random BS selection, distance based and greedy schemes. In the case of distance based sleep-mode algorithm it may seem that considering the fact that distance affected on parameters such as transmission power, the propagation and the path loss, it can be an appropriate indicator in the decision about which and how many BSs have to be switched OFF. However, the distance-based mechanism have major drawbacks. In this method it has been ignored that there may be numerous scenarios that indicative the same average distance. Suppose we have two cells that called $x$ and $y$. In the x-cell, can assume a case where some users are on the cell edge while others are close to their serving BS. In the latter case (cell y), all users are somewhere in the middle of a cell. When that both cells $x$ and $y$ have the same average distance between the BS and the user's within the cell, the average distance is not a suitable representative or indicator for BS ON/OFF switching. Actually while the two aforementioned cases have the same average distance, it might more feasible to off-load both users in scenario $y$ to other neighbor BS. On the other hand in scenario $x$, off-loading of user's that are very close to the BS to neighbor BS could prove to be quite costly due to its close proximity to the cell center.

In the case of the greedy-based BS switch OFF algorithm the distance of users from their serving BS are not considered. Overlook from the distance of users in some cases can be have serious costs. The next flaw in this approach is that the channel conditions are not considered. Also, all users are transferred to the next BS simultaneously and do not have user assignment.

Most optimization methods depend on algorithm control parameters that affects the performance and effectiveness of the algorithm. GA and PSO optimization algorithms are not excluded from it. If you closely examine these algorithms, you will get that GA requires the proper tuning of the specific parameters as population size, crossover probability, selection method and mutation rates and PSO algorithm likewise requires the variation of weight, the maximum value of velocity, learning factors, social and cognitive parameters. That's while the TLBO-based GOSBOURA does not require any algorithmic parameter adjustment, which gives it advantage over GA and PSO that are parameter dependent nonlinear optimization technique. TLBO utilizes two different phases, uses the mean value of the population to update the solution and implements greediness to accept a good solution that achieves the best answer. Reliability of the proposed scheme is significantly higher (lower standard deviation) than other schemes, especially proposed scheme with GA and random BS selection. It should be noted that because the distance based sleep- mode and greedy-based BS switch OFF algorithms follow the predefined process, the value of the standard deviation with 50 runs for each channel realization is zero. 
Table.3. Statistical properties of total cluster power as a random variable for three different network realizations, with 50 runs (50 random samples) for each channel realization

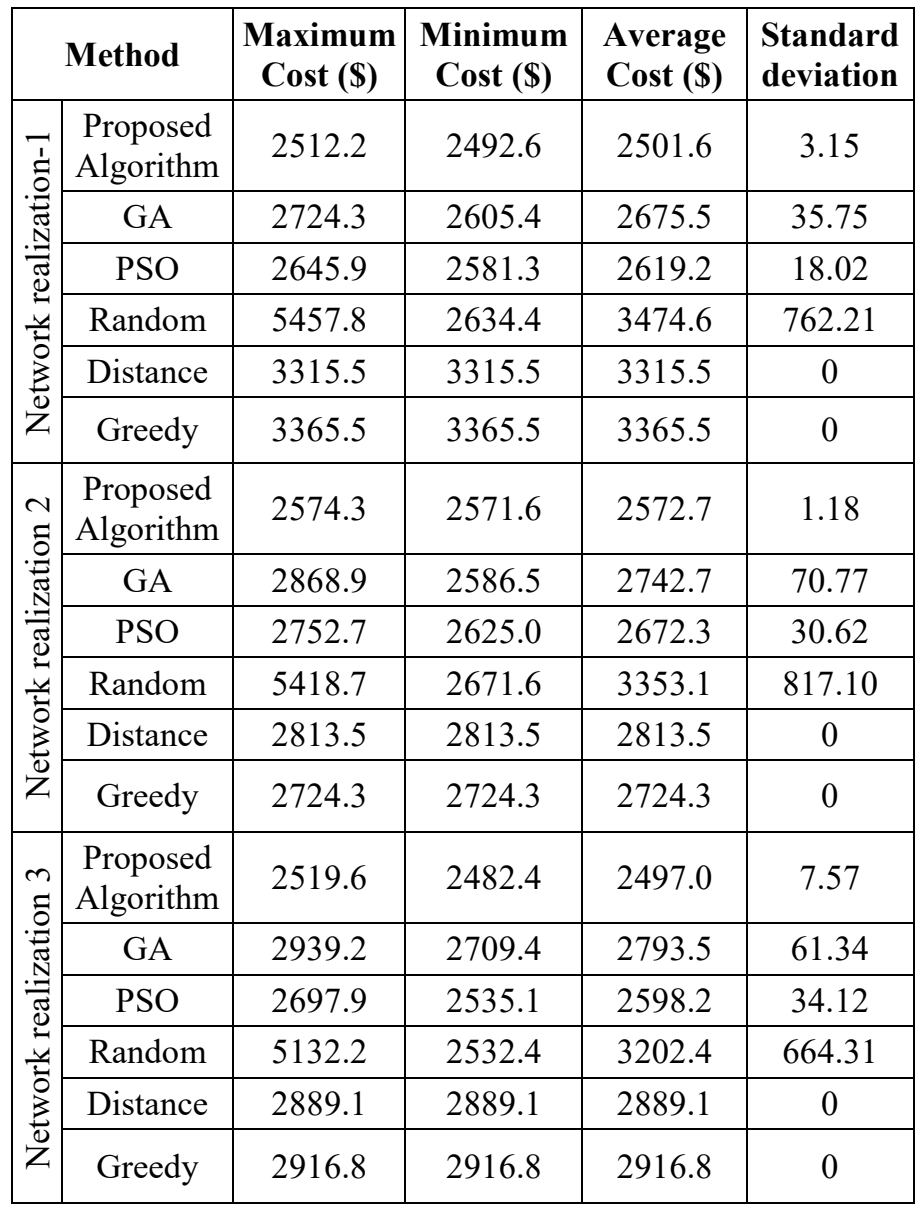

\subsection{EFFECT OF POPULATION SIZE}

TLBO is a population based heuristic search algorithm which implements a group of solutions to proceed to the optimum solution. In this regard, in the proposed method a list of unoccupied sub-channels will be provided for each BS that used to construct the initial population. In Table.4 effect of initial population size (step 3 of GOSBOURAO) on the proposed algorithm's performance has been investigated over the first cluster realization used to obtain results of Table.3. Increasing the initial population size of TLBO, when it is relatively small, has positive impact on network's total power consumption. However, its increase beyond some specific point does not affect the total power consumption. For the example shown in Table.4, initial population size of 200 is the critical point, where beyond that, larger population sizes do not achieve lower power. On the other hand, larger initial population size results in higher computational complexity.

\subsection{CONVERGENCE RATE}

Convergence rate of different algorithms has been compared by plotting total power consumption of the proposed scheme with different optimization techniques versus each technique's number of iterations, in Fig.7. TLBO has the highest convergence rate to the optimum solution compared to other schemes. Based on the results of the algorithms, TLBO converges in the $20^{\text {th }}$, PSO in the $32^{\text {th }}$ and GA in the $47^{\text {th }}$ iterations. Even at high number of iterations, GA and
PSO are not able to converge as close to the optimum point as TLBO does. TLBO algorithm by utilize the best solution in each iteration changes the solution available in the population, which results in a significant increase convergence rates.

Table.4. Effect of initial population size on the performance of GOSBOURAO

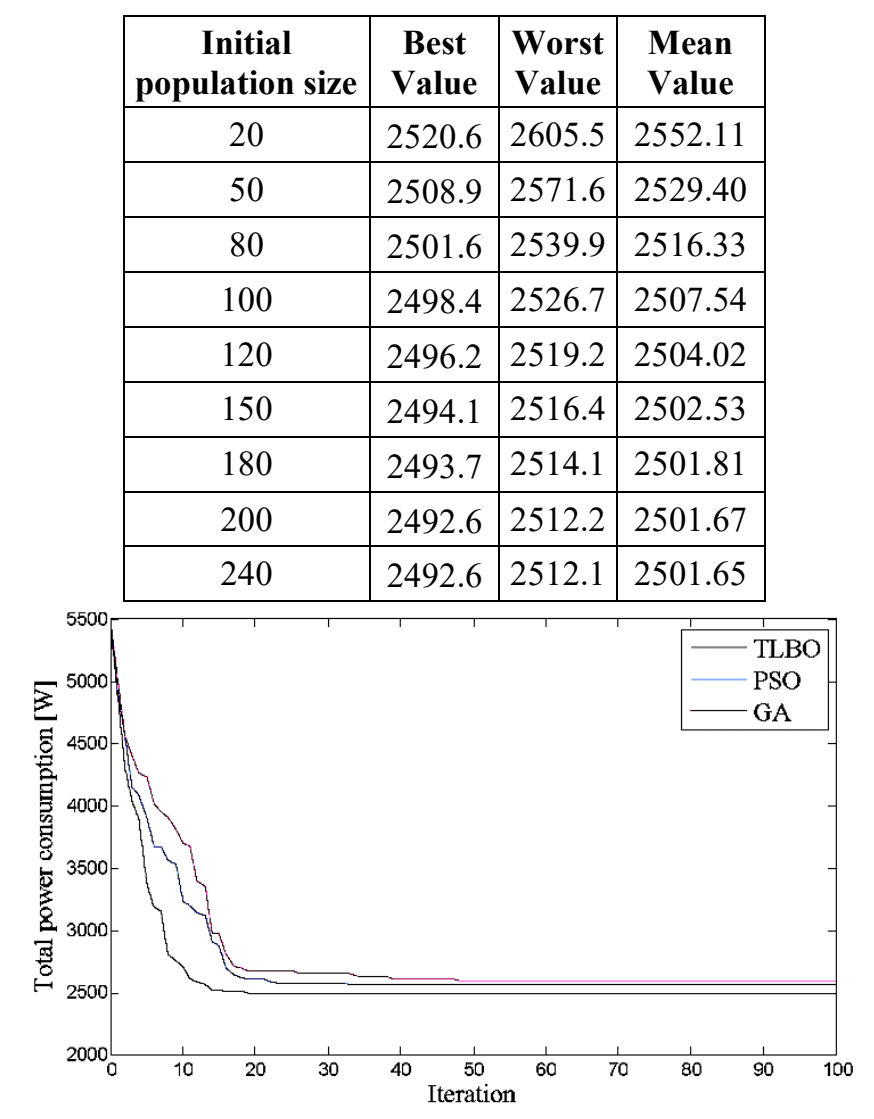

Fig.7. Convergence graphs of TLBO, PSO and Genetic Algorithm over the first cluster realization used to obtain results of Table. 3

\subsection{COMPLEXITY}

Finally, considering the results of the previous subsections, the initial population size has been set to 200 and CPU time of TLBO, PSO and GA for the third stage of BS switch OFF and over the first cluster realization used to obtain results of Table. 3 has been compared in Table5. All experiments have been carried out in Matlab V.7.14.0.739 on a Core i7 3.5-GHz personal computer with $24 \mathrm{~GB}$ of RAM memory. As the results of Table.5 show, TLBO achieves the lowest power consumption. It can be seen that the PSO algorithm has a higher execution speed than two other algorithms, TLBO and GA. The reason for this is that there is only one phase in the PSO algorithm and the number of times that the cost function is calculated is less. This is while TLBO uses two different phases, the "Teacher phase" and the "Learner phase". It is also observed that the genetic algorithm has a lower speed than the PSO and TLBO algorithms. As opposed to GA, PSO and TLBO use summation in vector form, and hence, are faster than GA. Also, the cross-over process of the genetic algorithm increases the execution time of the algorithm. 
Table.5. CPU Time Comparison

\begin{tabular}{|c|c|c|c|c|}
\hline Method & $\begin{array}{c}\text { Population } \\
\text { size }\end{array}$ & $\begin{array}{c}\text { Maximum } \\
\text { Iteration }\end{array}$ & $\begin{array}{c}\text { Power } \\
\text { (watt) }\end{array}$ & $\begin{array}{c}\text { Scaled CPU } \\
\text { time (min) }\end{array}$ \\
\hline GA & 200 & 20 & 2674.2 & 0.2840 \\
\hline PSO & 200 & 20 & 2601.6 & 0.1723 \\
\hline TLBO & 200 & 20 & 2492.6 & 0.2178 \\
\hline
\end{tabular}

\section{CONCLUDING AND REMARKS}

$\mathrm{EE}$ in cellular networks has become a growing concern not only in term of increase of greenhouse effects and environmental problems, but also especially for network operators due to operational expenditures (OPEX). Since BSs power consumption is the most important issue regard to the power of typical cellular networks, improving the EE of BSs is crucial for development of this networks, also reduce energy costs and environmental conditions improvement can be observed. This paper presented an efficient solution to the complex and nonlinear mix-integer programming problem of base station switch OFF and user subchannel allocation for minimizing cellular network's total power consumption in downlink OFDM-based networks. As the core of the proposed GOSBOURAO algorithm, TLBO was used as an efficient optimization tool. This algorithm has no user-defined parameter which makes it superior than earlier ones. We presented an algorithm for BS energy saving that encompasses both BS operation and user subcarrier-allocation. Simultaneous search for BS candidates to be switched OFF and user subcarrier assignment is a complex mix-integer and nonlinear optimization problem, thereby an efficient optimization technique are required to solve the aforementioned problem. The proposed scheme is compatible with the existing network and does not require any change in the current network structure, and as well as can be applied to any cellular architecture with any number of connected cells. Reliability of the proposed scheme is significantly higher than other schemes, which makes it a suitable solution for practical implementation. Numerical results indicated that the proposed scheme is able to reduce network's power consumption significantly compared to other existing sleep-mode-based algorithms. It was shown numerically that proposed scheme is robust in finding the optimum solution.

\section{REFERENCES}

[1] A. Abrol and R.K. Jha, "Power Optimization in 5G Networks: A Step towards Green Communication", IEEE Access, Vol. 4, pp. 1355-1374, 2016.

[2] Z. Hasan, H. Boostanimehr and V. Bhargava, "Green Cellular Networks: A Survey, Some Research Issues and Challenges", IEEE Communications Surveys and Tutorials, Vol. 13, No. 4, pp. 524-540, 2011.

[3] R. Mahapatra et al., "Energy Efficiency Trade-off Mechanism towards Wireless Green Communication: A Survey", IEEE Transactions on Wireless Communications, Vol. 18, No. 1, pp. 686-705, 2016.

[4] O. Arnold et al., "Power Consumption Modeling of Different Base station Types in Heterogeneous Cellular Networks", Proceedings of Future Network and Mobile Summit, pp. 1-8, 2010.
[5] A. Fehske, F. Richter and G. Fettweis, "Energy Efficiency Improvements through Micro Sites in Cellular Mobile Radio Networks", Proceedings of IEEE GLOBECOM Workshop, pp 1-5, 2009.

[6] L. Correia et al., "Challenges and Enabling Technologies for Energy Aware Mobile Radio Networks", IEEE Communications Magazine, Vol. 48, No. 11, pp. 66-72, 2010.

[7] C. Murthy and C. Kavitha, "A Survey of Green Base stations in Cellular Networks", International Journals of Computer Networks and Wireless Communications, Vol. 2, No. 2, pp. 232-236, 2012.

[8] P. Belotti et al., "Mixed-integer Nonlinear Optimization", http://homepages.cae.wisc.edu/ linderot/papers/Belotti-EtAl-12-TR.pdf.

[9] D. Ngo and T. Le-Ngoc, "Joint Sub Channel Assignment and Power Allocation for OFDMA Femtocell Networks", IEEE Transactions on Wireless Communications, Vol. 13, No. 1, pp. 342-355, 2014.

[10] C. Chang et al., "On Optimal Cell Activation for Coverage Preservation in Green Cellular Networks", IEEE Transactions on Mobile Computing, Vol. 13, No. 11, pp. 2580-2591, 2014.

[11] Y. Chen, W. Sheen and L. Wang, "Optimization of CyclicDelay Diversity Aided Frequency-Selective Scheduling in OFDMA Downlink Systems", IEEE Transactions on Vehicular Technology, Vol. 63, No. 4, pp. 1645-1659, 2014.

[12] Y. Chiu et al., "GGRA: a Feasible Resource-Allocation Scheme by Optimization Technique for IEEE 802.16 Uplink Systems", IEEE Transactions on Vehicular Technology, Vol. 59, No. 3, pp. 1393-1401, 2010.

[13] E. Danish et al., "Content-aware Resource Allocation in OFDM Systems for Energy Efficient Video Transmission", IEEE Transactions on Consumer Electronics, Vol. 60, No. 3, pp. 320-328, 2014.

[14] R. Elliott and W. Krzymien, "Downlink Scheduling via Genetic Algorithms for Multiuser Single-Carrier and Multicarrier MIMO Systems with Dirty Paper Coding”, IEEE Transactions on Vehicular Technology, Vol. 58, No. 7, pp. 3247-3262, 2009.

[15] J. Zhang et al., "Evolutionary-Algorithm-Assisted Joint Channel Estimation and Turbo Multiuser Detection/Decoding for OFDM/SDMA", IEEE Transactions on Vehicular Technology, Vol. 63, No. 3, pp. 1204-1222, 2014.

[16] H. Lang, S. Lin and W. Fang, "Subcarrier Pairing and Power Allocation with Interference Management in Cognitive Relay Networks Based on Genetic Algorithms", IEEE Transactions on Vehicular Technology, Vol. 65, No. 9, pp. 7051-7063, 2015.

[17] N. Sharma and A.S. Madhukumar, "Genetic Algorithm Aided Proportional Fair Resource Allocation in Multicast OFDM Systems", IEEE Transactions on Broadcasting, Vol. 61, No. 1, pp. 16-29, 2015.

[18] R. Andreotti et al., "Resource Allocation via Max-Min Good Put Optimization for BIC-OFDMA Systems", IEEE Transactions on Communications, Vol. 64, No. 6, pp. 24122426, 2016.

[19] K. Bagadi and S. Das, "Neural Network-based Adaptive Multiuser Detection Schemes in SDMA-OFDM System for 
Wireless Application", Neural Computing and Applications, Vol. 23, No. 3-4, pp. 1071-1082, 2013.

[20] D. Astely et al., "A Future Radio Access Framework", IEEE Journal on Selected Areas in Communications, Vol. 24, No. 3, pp. 693-706, 2006.

[21] Rysavy Research, "Transition to 4G: 3GPP broadband evolution to IMT advanced", Available at: https://www.ic.gc.ca/eic/site/smt-gst.nsf/vwapj/dgso-00110-3GAmericas-atatchment.pdf/\$FILE/dgso-001-103GAmericas-atatchment.pdf.

[22] W. Rhee and J. Cioffi, "Increase in Capacity of Multiuser OFDM System using Dynamic Subchannel Allocation", Proceedings of IEEE 51 $1^{\text {st }}$ Vehicular Technology Conference, pp. 1085-1089, 2000.

[23] F. Shams, G. Bacci and M. Luise, "A Survey on Resource Allocation Techniques in OFDM (A) Networks", Computer Networks, Vol. 65, pp. 129-150, 2014.

[24] R. Bolla et al., "Energy Efficiency in the Future Internet: A Survey of Existing Approaches and Trends in Energy-aware Fixed Network Infrastructures", IEEE Communications Surveys and Tutorials, Vol. 13, No. 2, pp. 223-244, 2011.

[25] T. Han and N. Ansari, "On Greening Cellular Networks via Multicell Cooperation”, IEEE Wireless Communications, Vol. 20, No. 1, pp. 82-89, 2011.

[26] B. Badic et al., "Energy Efficient Radio Access Architectures for Green Radio: Large Versus Small Cell Size Deployment", Proceedings of IEEE $70^{\text {th }}$ International Conference on Vehicular Technology, pp. 1-5, 2009.

[27] T. Le and M. Nakhai, "Possible Power-Saving Gains by Dividing a Cell into Tiers of Smaller Cells", Electronics Letters, Vol. 46, No. 16, pp. 1163-1165, 2010.

[28] B. Badic et al., "Effect of the Base station Antenna Beam Tilting on Energy Consumption in Cellular Networks", Proceedings of IEEE $72^{\text {nd }}$ International Conference on Vehicular Technology, pp. 1-5, 2010.

[29] F. Cao and Z. Fan, "The Tradeoff between Energy Efficiency and System Performance of Femtocell Deployment", Proceedings of IEEE $7^{\text {th }}$ International Symposium on Wireless Communication Systems, pp. 315-319, 2010.

[30] T. Tamadoni, M. Eslami, Sh. Jam and D. Davarpanah, "A Novel Antenna Allocation Technique for Green Single Cell MIMO and MIMO-CoMP Downlink Transmission", AEUInternational Journal of Electronics and Communications, Vol. 80, pp. 59-66, 2017.

[31] M.A. Marsan et al., "Optimal Energy Savings in Cellular Access Networks", Proceedings of IEEE International Conference on Communications, pp. 1-5, 2009.

[32] E. Oh and B. Krishnamachari, "Energy Savings through Dynamic Base station Switching in Cellular Wireless Access Networks", Proceedings of IEEE International Conference on Global Telecommunications, pp. 1-5, 2010.

[33] A. Hajijamali Arani et al., "Distributed ON/OFF Switching and Dynamic Channel Allocation: Decreasing Complexity and Improving Energy Efficiency", Transactions on Emerging Telecommunications Technologies, Vol. 28, No. 12, pp. 1-12, 2015.

[34] K. Samdanis et al., "Self-organized Energy Efficient Cellular Networks", Proceedings of IEEE $21^{\text {st }}$ International Symposium on Personal Indoor and Mobile Radio Communications, pp. 1665-1670, 2010.
[35] A. Bousia et al., "Green Distance-Aware Base Station Sleeping Algorithm in LTE Advanced", Proceedings of IEEE International Conference on Communications, pp. 1347-1351, 2012.

[36] C. Xiong et al., "Energy-Efficient Resource Allocation in OFDMA Networks", IEEE Transactions on Communications, Vol. 60, No. 12, pp. 3767-3778, 2015.

[37] S. Elayoubi, L. Saker and T. Chahed, "Optimal Control for Base station Sleep Mode in Energy Efficient Radio Access Networks", Proceedings of IEEE Conference on INFOCOM, pp. 106-110, 2011.

[38] Z. Niu et al., "Cell Zooming for Cost-efficient Green Cellular Networks", IEEE Communications Magazine, Vol. 48, No. 11, pp. 74-79, 2010.

[39] S. Bhaumik et al., "Breathe to Stay Cool: Adjusting Cell sizes to reduce Energy Consumption", Proceedings of $1^{\text {st }} A C M$ SIGCOMM Workshop on Green Networking, pp. 41-46, 2010.

[40] G. Koutitas, "Green Network Planning of Single Frequency Networks", IEEE Transactions on Broadcasting, Vol. 56, No. 4, pp. 541-550, 2010.

[41] H. Ghazzai et al., "Optimized Smart Grid Energy Procurement for LTE Networks using Evolutionary Algorithms", Transactions on Vehicular Technology, Vol. 63, No. 9, pp. 4508-4519, 2014.

[42] L. Xiang et al., "Adaptive Traffic Load-Balancing for Green Cellular Networks", Proceedings of IEEE $22^{\text {nd }}$ International Symposium on Personal Indoor and Mobile Radio Communications, pp. 41-45, 2011.

[43] D. Goldberg, "Genetic Algorithms in Search, Optimization and Machine Learning”, Addison Wesley Publisher, 1989.

[44] M. Clerc, "Particle Swarm Optimization", Available at: https://kamenpenkov.files.wordpress.com/2016/01/pso-mclerc-2006.pdf

[45] D. Karaboga, "An Idea Based on Honey Bee Swarm for Numerical Optimization”, Technical Report, Department of Computer Engineering, Erciyes University, 2005.

[46] M. Dorigo and C. Blum, "Ant Colony Optimization: A Survey", Theoretical Computer Science, Vol. 344, No. 2-3, pp. 243-278, 2011.

[47] K.S. Lee and Z.W. Geem, "A New Meta-Heuristic Algorithm for Continuous Engineering Optimization: Harmony Search Theory and Practice", Computer Methods in Applied Mechanics and Engineering, Vol. 194, No. 3638, pp. 3902-3933, 2005.

[48] A. Ahrari and A.A. Atai, "Grenade Explosion Method a Novel Tool for Optimization of Multimodal Functions", Applied Soft Computing, Vol. 10, No. 4, pp. 1132-1140, 2010.

[49] R.V. Rao, V.D. Kalyankar and G. Waghmare, "Parameters Optimization of Selected Casting Processes using Teaching Learning based Optimization Algorithm", Applied Mathematical Modelling, Vol. 38, No. 23, pp. 5592-5608, 2014.

[50] M.H. Khooban, "Design an Intelligent Proportional-Derivative Feedback Linearization Control for Nonholonomic-Wheeled Mobile Robot", Journal of Intelligent \& Fuzzy Systems, Vol. 26, No. 4, pp. 1833-1843, 2014.

[51] R.V. Rao, V.J. Savsani and D.P. Vakharia, "TeachingLearning-based Optimization: A Novel Method for Constrained Mechanical Design Optimization Problem", Computer-Aided Design, Vol. 43, No. 3, pp. 303-315, 2011. 